\title{
Spontaneous Electrical Activity in the Human Fetal Cortex In Vitro
}

\author{
Anna R. Moore, Wen-Liang Zhou, Igor Jakovcevski, Nada Zecevic, and Srdjan D. Antic \\ Department of Neuroscience, University of Connecticut Health Center, Farmington, Connecticut 06030
}

Our knowledge about the developing human cerebral cortex is based on the analysis of fixed postmortem material. Here we use electrical recordings from unfixed human postmortem tissue to characterize the synaptic physiology and spontaneous network activity of pioneer cortical neurons ("subplate neurons"). Our electrophysiological experiments show that functional glutamate or GABA ionotropic receptors are expressed on human subplate (SP) neurons as early as 20 gestational weeks. Extracellular (synaptic) stimulations evoked postsynaptic potentials in a very small fraction of SP neurons, suggesting that functional synaptic contacts are rare at midgestation. Although synaptic inputs were scarce, we regularly observed spontaneous (unprovoked) electrical activity among human SP neurons, comprised of sustained plateau depolarizations and bursts of action potential firing, which resembled cortical UP and DOWN states in the adult neocortex. Plateau depolarizations and bursts of action potential firing are thought to depend on the mature morphology and physiology of adult cortical network. However, our current data reveal that similar cortical rhythm is generated by a very immature ensemble of human fetal neurons. In the relative absence of sensory inputs, as in development in utero, or in slow-wave sleep (i.e., throughout the entire lifespan), the spontaneous slow oscillatory pattern (UP and DOWN states) is a fundamental aspect of human cortical physiology.

\section{Introduction}

Much of our knowledge about human fetal cortex is based on the analysis of fixed postmortem material (anatomy and histochemistry), while physiological characterization of young human neurons is currently unavailable. This is unfortunate, because a growing body of animal research indicates that electrical activity of early neurons is essential for proper brain development (Moody and Bosma, 2005). For example, some elaborate features of the adult cortex (e.g., ocular dominance columns) exist in the embryonic brains of experimental animals, and therefore activity-dependent processes must operate before the onset of sensory-evoked activity (Rakic, 1976). In support of this notion, correlated spontaneous activity has been observed in the rodent cortex several days before birth (Corlew et al., 2004; Allène et al., 2008) and several days after birth (Yuste et al., 1992; Garaschuk et al., 2000), while the rodent cortex is still undergoing rapid development. Does the human cerebral cortex generate spontaneous electrical activity before arrival of sensory inputs (before birth)? If yes, what neurons support electrical oscillations?

We tackled this question by focusing on one prominent anatomical region, the subplate (SP) zone, which emerges during early cortical development and governs the pathfinding of in-

Received July 26, 2010; revised Nov. 28, 2010; accepted Dec. 1, 2010.

This work was supported by a National Institutes of Health (NIH) training grant to A.R.M. and NIH R01 grants to N.Z. and S.D.A. Human fetal tissue was obtained from B. Poulos at the Albert Einstein College of Medicine, Tissue Repository, The Bronx, NY. We thank Debbie Bishop, Doug Oliver, and Dick Mains for access to Department of Neuroscience core equipment.

Correspondence should be addressed to Srdjan D. Antic, Department of Neuroscience, University of Connecticut Health Center, Farmington, CT 06030. E-mail: antic@neuron.uchc.edu.

DOI:10.1523/JNEUROSCI.3886-10.2011

Copyright $\odot 2011$ the authors $\quad 0270-6474 / 11 / 312391-08 \$ 15.00 / 0$ coming axonal projections (Ghosh et al., 1990; Kostovic and Rakic, 1990). This zone contains some of the earliest born (pioneer) neurons in the cerebral cortex (Luskin and Shatz, 1985), and therefore, it is likely to be the site of early electrical activity. Electrical recordings were carried out in an acute human fetal brain slice preparation to characterize spontaneous (unprovoked) membrane potential oscillations. In each tissue specimen tested [ $n=5$ human subjects, fetal age 20-21 gestational weeks (gw)], we found a substantial number of cortical SP neurons that exhibited robust spontaneous activity, comprised of action potentials (APs) riding atop large plateau depolarizations. The periods of such spontaneous (unprovoked) outbursts of electrical activity were interrupted by long and irregular periods of silence, resembling "tracé discontinu" in electroencephalogram (EEG) recordings from preterm infants (Lamblin et al., 1999; André et al., 2010). Furthermore, individual outbursts of electrical activity among cortical subplate neurons in the fetal cortex in vitro resembled cortical UP states documented in the adult brain during anesthesia (Cowan and Wilson, 1994) and deep sleep (Steriade et al., 2001; Chauvette et al., 2010). Our data thus suggest that an inherent physiological property of the human cerebral cortex is a propensity to generate spontaneous activity (oscillation between DOWN and UP states) in the relative absence of sensory experience [e.g., before birth (Lamblin et al. 1999; present data) and during sleep (Marshall et al., 2006)].

\section{Materials and Methods}

Human tissue

Postmortem human fetal brain tissue ( $n=5$ cases), within the second trimester of gestation $[20(n=2$; female $)$ and $21(n=3 ; 2$ females and 1 unknown) gestational weeks], was obtained from the Tissue Repository of the Albert Einstein College of Medicine. Tissue fragments were exam- 
ined only with proper parental consent and the approval of the Ethics Committees at both Universities. Apart from gestational age and sex, no other information was received. Tissue specimens were transferred on ice in oxygenated HBSS (travel time was $\sim 3 \mathrm{~h}$ ). In the previous study, we reported a travel time of $\sim 2 \mathrm{~h}$. Lately, we have been spending at least $2.5 \mathrm{~h}$ on the road, and therefore $3 \mathrm{~h}$ is a more accurate estimate.

Due to the postmortem time delay of $15 \mathrm{~min}$ and long travel time of $2-3 \mathrm{~h}$, followed by an additional 30 min needed for making the acute slices, it is predicted that a majority of human fetal neurons would be unable to withstand such extreme conditions. But young neurons are resilient to hypoxic situations and metabolic stress (Xia and Haddad, 1999; Zhang et al., 2001). The amount of cell death present in fetal brain slices following extraction has previously been tested using the in situ TUNEL method, which detects early DNA fragmentation in apoptotic cells (Rakic and Zecevic, 2000). Based on Neurolucida drawings illustrating the TUNEL $(+)$ nuclei, the majority of cell death is limited to the proliferative regions of the ventricular and subventricular zones with a maximum apoptotic index of $4.7 \pm 0.7$ TUNEL(+) nuclei per $10^{6} \mu \mathrm{m}^{3}$ (Rakic and Zecevic, 2000). Overall the majority of cells present in the acute brain slice are still viable. In addition to the TUNEL assays performed on previous human material, one can also verify the viability of human fetal cells based on their appearance under differential interference contrast (DIC) video microscopy (Fig. $1 B$, SP neuron), and by detecting a negative resting membrane potential (more negative than $-40 \mathrm{mV}$ ) and high input resistance (Moore et al., 2009).

\section{Whole-cell patch-clamp recordings}

All physiological recordings were done on the day of tissue collection. Brain slices, $500 \mu \mathrm{m}$ to $1 \mathrm{~mm}$ thick, obtained from the medial telencephalic wall of the occipital lobe, were sectioned by hand while kept on ice-cold artificial CSF (ACSF) solution. The ACSF contained (in mM) 125 $\mathrm{NaCl}, 2.3 \mathrm{KCl}, 26 \mathrm{NaHCO}_{3}, 2 \mathrm{MgSO}_{4}, 1.26 \mathrm{KH}_{2} \mathrm{PO}_{4}, 2 \mathrm{CaCl}_{2}$, and 20 glucose, $\mathrm{pH} 7.3$ (osmolality $=310 \mathrm{mOsm} / \mathrm{kg}$ ). Slices were incubated at $37^{\circ} \mathrm{C}$ for $0.5 \mathrm{~h}$ and then stored at room temperature before recordings. Patch-clamp recordings were performed as previously described (Moore et al., 2009). Patch pipettes (7-12 M $\Omega$ ) were filled with an intracellular solution containing (in $\mathrm{mM}$ ) $135 \mathrm{~K}$-gluconate, 10 HEPES, $2 \mathrm{MgCl}_{2}, 3$ ATP- $\mathrm{Na}_{2}, 0.3$ GTP-Na $\mathrm{Na}_{2}$, and 10 phosphocreatine $\mathrm{Na}_{2}, \mathrm{pH} 7.3$ adjusted with $\mathrm{KOH}$ (osmolality $=300 \mathrm{mOsm} / \mathrm{kg}$ ). For fluorescent identification of cellular morphology, either sulforhodamine 101 or Alexa Fluor 594 (Invitrogen) or Lucifer yellow (Sigma) was added to the intracellular solution at concentrations between 30 and $50 \mu \mathrm{M}$. Membrane potential values were corrected for the liquid junction potential $-10.4 \mathrm{mV}$.

\section{Recordings of spontaneous activity}

Upon verification of repetitive AP firing (Fig. $1 E$ ), we proceeded to record spontaneous electrical activity (Figs. 2, 3). Voltage monitoring was performed with a holding current $(-10.4 \pm 0.38 \mathrm{pA})$ added to keep each neuron at around $-60 \mathrm{mV}$. Once the recording of spontaneous activity started, the intensity of bias current was kept unchanged.

\section{Synaptic stimulation}

Glass micropipettes (4-7 M $\Omega$ ) were filled with ACSF, attached to the motorized micromanipulator and placed within $50-150 \mu \mathrm{m}$ of the SP neuron somata. Electrical shocks were generated by a computer-driven stimulus isolation unit, in constant-current mode, synaptic pulse duration $100-200 \mu \mathrm{s}$, and intensity range $10-150 \mu \mathrm{A}$. The stated current amplitude values represent the settings on the instrument control panel. The identical apparatus (same rig, same electrode size and material, and same pulse generator) invariably triggers postsynaptic potentials in rat or mouse cortical slice, when the current amplitude was set at 10-80 $\mu \mathrm{A}$.

\section{Glutamate microiontophoresis}

Sharp glass pipettes $(40 \pm 10 \mathrm{M} \Omega)$ were filled with $200 \mathrm{~mm}$ sodium glutamate, $\mathrm{pH} 9$, attached to the motorized micromanipulator, and positioned within $30-150 \mu \mathrm{m}$ of the cell soma using infrared videomicroscopy. An Iso-Flex (A.M.P.I.) stimulus isolation unit, controlled by data acquisition software Clampex 9.2 (Molecular Devices), was used to deliver (5 ms duration and 1.0-3.0 $\mu \mathrm{A}$ amplitude) constant negative current pulses. A minus sign is omitted in the text.

\section{GABA pressure ejections}

Glass micropipettes (4-7 M $\Omega$ ) were filled with $25 \mathrm{~mm} \mathrm{GABA}$, attached to the motorized micromanipulator and placed within $150 \mu \mathrm{m}$ of the SP neuron somata. A computer-driven picospritzer was used to pressure eject GABA near the cell $(30-150 \mu \mathrm{m})$. Pulse duration and pressure intensities varied in the range 500-1000 $\mathrm{ms}$ and $20-60 \mathrm{mbar}$, respectively. In some instances, no pressure was needed to detect GABA action on SP neurons. For example, in the experiment depicted in Figure $4 G$, the SP cell was inhibited as soon as the tip of the GABA pipette was brought within $50 \mu \mathrm{m}$ from the cell body. The effect of GABA was stopped by moving the GABA pipette away (Fig. 4G, GABA).

\section{Immunohistochemistry and imaging}

Following electrophysiological recordings, tissue slices were placed in $4 \%$ paraformaldehyde at $4^{\circ} \mathrm{C}$ overnight and then resectioned to $20 \mu \mathrm{m}$ thick slices. Sections were mounted onto gelatin-coated glass coverslips, permeabilized, and incubated in Hoechst (dilution 1:2000) for $1 \mathrm{~h}$. Images were taken on an Axiovert microscope (Zeiss).

\section{Data analysis}

Data analysis was performed offline in Clampfit 9.2, Excel, and SigmaStat 3.2. Summary data are expressed as mean \pm SEM. 
A
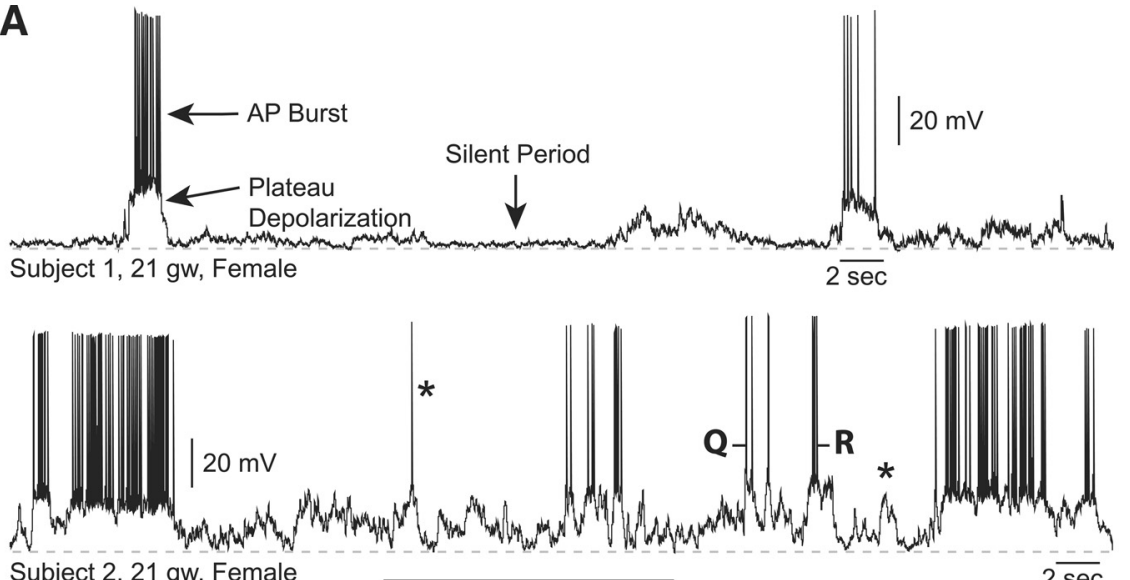

Subject 2, $21 \mathrm{gw}$, Female

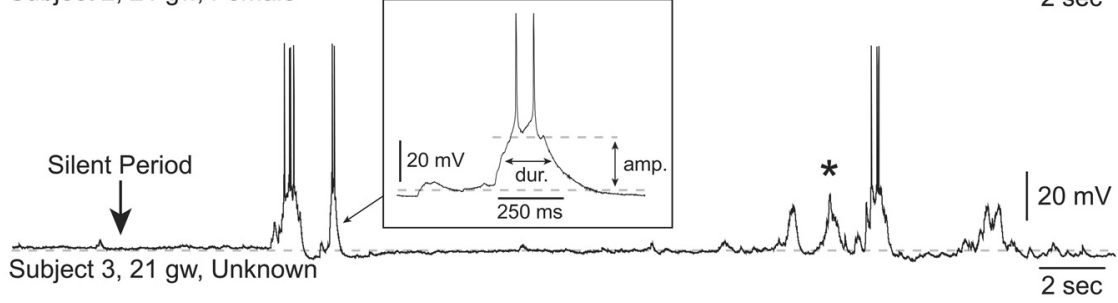

B

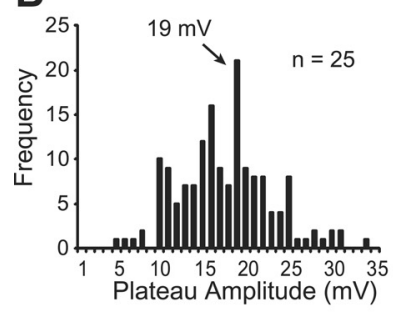

C
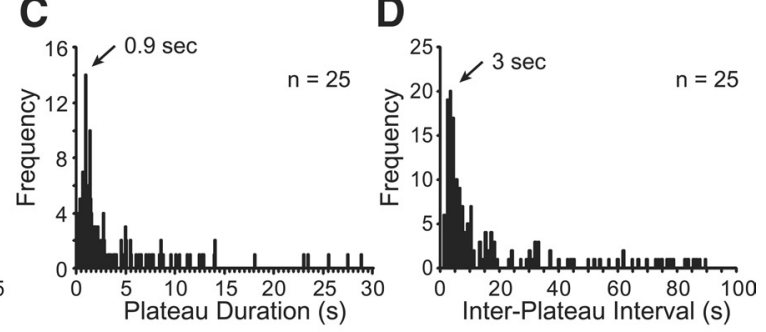

E
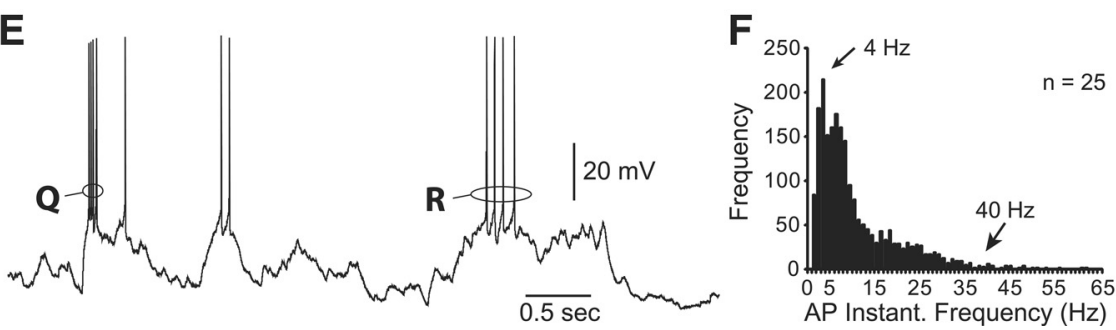

Figure 2. Spontaneous electrical activity before birth. $\boldsymbol{A}$, Spontaneously active SP neurons from three human subjects. Dashed lines, Resting potentials ( $-60,-62$, and $-63 \mathrm{mV}$, respectively). Asterisks indicate UP states with one or zero action potentials (APs). Inset, Duration (dur.) and amplitude (amp.) of an UP state. Parameters of UP states ( $n=162$ events, 25 neurons) are plotted using frequency histograms in $\boldsymbol{B}-\boldsymbol{D}$ and $\boldsymbol{F}$. $\boldsymbol{B}$, The most frequent (mode) plateau amplitude was $19 \mathrm{mV}$. $\boldsymbol{C}$, The most frequent plateau duration was 0.9 s. D, Variability in time interval between successive UP states. The most common interval was 3 s. $\boldsymbol{E}$, Expanded fragment of trace 2 shown in $\boldsymbol{A}$. The high-frequency-accommodating AP burst " $Q$ " is followed by low-frequencyregular firing AP burst "R." $F$, During plateau depolarizations SP neurons experience a wide range of AP firing frequencies. The most common AP firing rate was $4 \mathrm{~Hz}$.

Voltage dwelling. An all-points histogram (Fig. 3B) was made in Clampfit 9.2. The resting membrane potential was set to $0 \mathrm{mV}$, and bin size to $1 \mathrm{mV}$.

Plateau amplitude. The "amplitude of UP state" refers to the amplitude of the slow component of an UP state event. In the present study, the plateau amplitude was measured from the baseline just before UP state to the arbitrary horizontal line (supplemental Fig. S2 B, available at www. jneurosci.org as supplemental material, inset). The horizontal lines were positioned arbitrarily (manually) for each plateau event. Action potentials were ignored in this quantification according to previously established strategies (Stern et al., 1997; Chauvette et al., 2010). If we dismiss action potentials, the membrane potential is restricted within a narrow membrane potential range during the UP state (Figs. 2, 3). Therefore, an arbitrary horizontal line (supplemental Fig. S2 B, available at www.jneurosci.org as supplemental material, inset) approximates the mean of subthreshold membrane potential fluctuations during the plateau phase.

Plateau duration. Event duration was measured at the $3 \mathrm{mV}$ (arbitrary) threshold potential from baseline. Plateau durations thus reflect a time period between a point where the rising phase of the plateau event crosses the 3 $\mathrm{mV}$ threshold and the point at which the falling phase of plateau potential crosses the same 3 $\mathrm{mV}$ threshold.

Interplateau interval. Interevent interval was the time spent from the onset of one event to the onset of the following event measured at 3 $\mathrm{mV}$ above resting.

Instantaneous frequency. Instantaneous frequency was measured for action potentials occurring during the UP state, by dividing $1 \mathrm{~s}$ by the time interval between the rising phases of two subsequent action potentials.

\section{Results}

All recordings were made in the subplate zone of the human fetal occipital cortex at 20 and $21 \mathrm{gw}$ (Fig. 1A). Subplate neurons were identified based on four criteria: (1) location within the SP zone (Fig. 1A); (2) characteristic appearance of the cell body in infrared DIC video microscopy [typically a large diameter (Fig. $1 B$, SP Neuron); quite often, SP cells had one primary neurite extending toward the pia or subventricular zone (SVZ), and multiple secondary neurites (Fig. 1C)]; (3) large sodium currents (peak $>700$ pA) (Fig. 1D); and (4) repetitive AP firing upon current injection (Fig. $1 E)$. Only those neurons meeting the four criteria were used in the current study ( $n=$ 49 neurons, 5 human subjects).

\section{Spontaneous electrical activity}

Uninterrupted recording sessions from fetal cells $(>5 \mathrm{~min}$ ) revealed that in the second trimester of gestation $(20-21 \mathrm{gw})$, SP neurons of the developing human cortex generate spontaneous (unprovoked) electrical activity in vitro (Fig. $2 A$ ). Note that the extracellular fluid (see Materials and Methods) contained calcium concentrations typically used in slice recordings (2 mM), as opposed to $1.2 \mathrm{~mm}$, used to induce spontaneous activity in adult brain slices (Sanchez-Vives and McCormick, 2000). The most prominent feature of human SP spontaneous activity was a sustained (plateau) depolarization crowned by bursts of full-size APs (Fig. $2 A$, AP Burst). The average amplitude of the plateau was $17.8 \pm$ $0.5 \mathrm{mV}$ (Fig. $2 \mathrm{~B}$ ), which is comparable to muscarine-induced UP states in the neonatal rodent SP (Hanganu et al., 2009) and cortical UP states in adult rodent cortex (Cowan and Wilson, 1994). The durations of UP states in human SP neurons varied greatly (200 ms-30 s), with the greatest frequency of events found at 900 ms (Fig. 2C). There was an apparent lack of rhythmicity in the occurrence of SP UP states (Fig. 2A). An isolated UP state, or a 

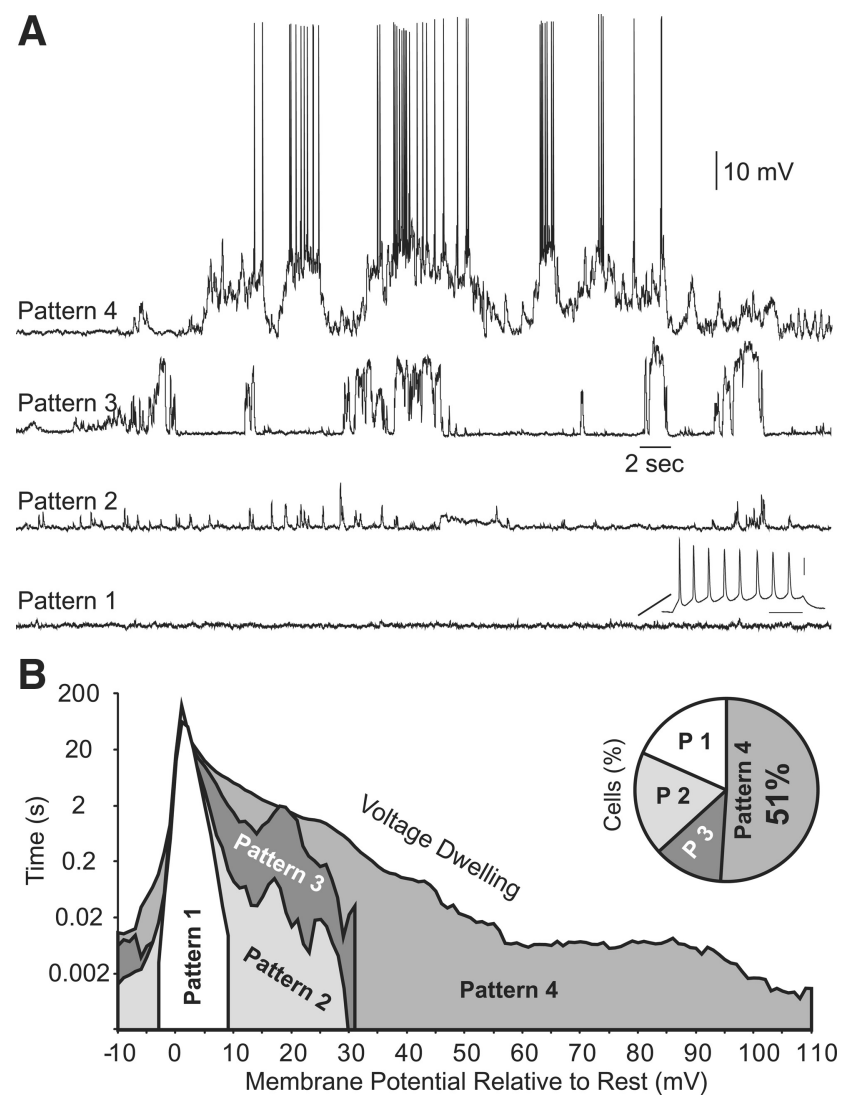

Figure 3. Four patterns of spontaneous electrical activity in human SP neurons. $A$, Human SP neurons display four patterns of spontaneous electrical activity (Pattern 1-4). B, All-points histogram reveals the average total amount of time that cells in four groups (Pattern 1-4) spend at a particular membrane potential (Voltage Dwelling). Resting potential is set to $0 \mathrm{mV}$. Voltage bin $=1 \mathrm{mV}$. Inset, Percentage of SP neurons belonging to each pattern $(\mathrm{P})$. The total number of neurons in the dataset is 49 from five human subjects, $20-21$ gestational weeks.

group of successive UP states, emerged sporadically and unpredictably during the course of each recording session (supplemental Fig. S2, available at www.jneurosci.org as supplemental material). It was not unusual to have long ( $>20 \mathrm{~s})$ periods of silence (Fig. $2 \mathrm{~A}$, Silent Period). The most frequent interval between UP states was $3 \mathrm{~s}$; however, there were many intervals that were over one order of magnitude greater (Fig. 2D; supplemental Fig. S2 $B$, available at www.jneurosci.org as supplemental material). During a plateau depolarization, APs were observed to fire in isolation (Fig. $2 A,{ }^{*}$ ) or within bursts of APs (Fig. $2 A$, AP Burst), thus encompassing a very wide range of frequencies (Fig. $2 F$ ). Quite often, the successive UP states were accompanied by AP bursts that differed in both the frequency and dynamics of the train [e.g., high vs low frequency; accommodating vs regular firing (Fig. 2E, Q vs R)]. The peak firing frequencies of human SP neurons $(40-60 \mathrm{~Hz})$ were comparable to those reported in early postnatal rodent SP neurons (Luhmann et al., 2009).

\section{Four patterns of electrical activity}

Recordings obtained in 49 SP neurons from five human subjects (20-21 gw) suggested the existence of four basic patterns (patterns 1-4) (Fig. 3A). In 9 of 49 cells, over the course of $10 \mathrm{~min}$, there were zero membrane potential transients that resembled synaptic inputs with peak amplitudes greater than $3 \mathrm{mV}$ (Fig. $3 \mathrm{~A}$, Pattern 1). In each of these 9 cells, direct current depolarizations evoked full-size repetitive APs (Fig. 3A, Pattern 1, inset), ensuring their health. "Pattern 2" SP neurons (9/49) displayed sharp de- polarization events of relatively short duration ( $\leq 0.5 \mathrm{~s})$ and mean peak amplitude of $5.5 \pm 0.2 \mathrm{mV}$ (Fig. 3A, Pattern 2). The third category of spontaneous electrical activity (Fig. $3 A$, Pattern $3)$ was comprised of long-lasting $(>1 \mathrm{~s})$ large-amplitude $(>10$ $\mathrm{mV})$ depolarization plateaus lacking AP firing $(n=6 / 49)$. The mean amplitude and duration of spontaneously occurring spikeless plateau depolarizations were $14 \pm 0.6 \mathrm{mV}$ and $2.1 \pm 0.4 \mathrm{~s}$, respectively. The most prevalent pattern of spontaneous electrical activity recorded in human SP neurons at midgestation $(20-21 \mathrm{gw})$ was the one with complete UP states, where APs fired atop large and long-lasting depolarization plateaus (Fig. $3 A$, Pattern 4). Pattern 4 activity was documented in $51 \%$ of recorded SP neurons ( $n=25 / 49)$. The "all-points histogram" (Fig. 3B, Voltage Dwelling) shows that human SP neurons with pattern 1 spontaneous activity spend most of their time close to resting potential (set to $0 \mathrm{mV}$ ) and have the smallest range of voltage excursions (Fig. 3B, Pattern 1). The voltages of pattern 2 and pattern 3 groups were similar in range, except neurons with pattern 3 activity spend considerably more time between 10 and $30 \mathrm{mV}$ above resting. Only SP neurons with pattern 4 activity reached voltages over $+30 \mathrm{mV}$ due to APs occurring during plateau depolarizations (Fig. 3B). ANOVA analysis performed on four subsets of human SP neurons (patterns 1-4) failed to detect any statistically significant differences in respect to (A) membrane potential; (B) membrane input resistance; or $(\mathrm{C})$ number of APs evoked by standard current pulse ( $p>0.05$ ) (supplemental Fig. S3A, available at www.jneurosci.org as supplemental material). In summary, although we distinguish four basic patterns of spontaneous electrical activity (supplemental Figs. S4, S5, available at www. jneurosci.org as supplemental material), our present data do not support more than one SP cell type (Luhmann et al., 2009). We propose that the same human SP cell type may alternate between two or more patterns of spontaneous activity (Fig. 2A, Silent period). Each human subject in the present study contained SP neurons characterized by more than one dominant activity pattern (supplemental Table 1, available at www.jneurosci.org as supplemental material).

\section{Human SP receive scarce synaptic input}

To check for existence of functional synaptic contacts onto human SP neurons, we delivered extracellular electric shocks in three characteristic locations: (1) between the SP neuron and SVZ (Fig. 4A, below), (2) between the SP neuron and cortical plate (CP) (above), or (3) lateral to the cell body (lateral). Despite multiple stimulation sites per neuron and a range of stimulus intensities $(10-150 \mu \mathrm{A})$, the majority of SP neurons $(65 \% ; n=$ $15 / 23$ ) were unable to generate a single postsynaptic potential (Fig. 4B). However, in 5 of 23 (22\%) SP neurons, we did observe synaptically evoked depolarizing potentials (Fig. 4C,D). These EPSP-like potentials were variable in amplitude and duration, unreliable (high incidence of failures), and difficult to find with blind synaptic stimulation. Synaptically evoked hyperpolarizing transients (IPSP-like), found in 3 of 23 (13\%) cells, were more regular and more uniform in amplitude and duration. IPSP-like potentials had a mean amplitude of $10.2 \pm 0.7 \mathrm{mV}$ and long durations ( $2.1 \pm 0.4 \mathrm{~s} ; n=3,5$ repetitions per cell) (Fig. $4 E)$ and were blocked by bath application of bicuculline $(20 \mu \mathrm{M}, n=1)$, indicating their GABAergic origin.

So far, our experimental data suggested that at midgestation (20-21 gw), a significant fraction of human SP cells are void of physiologically competent synaptic connections (Fig. 4C). One, out of many, possible explanations for the discrepancy between our physiological data (lack of functional synapses) and the ex- 
A Stim. Paradigm
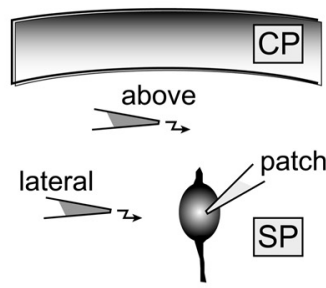

below $\_$Syn. Stim.

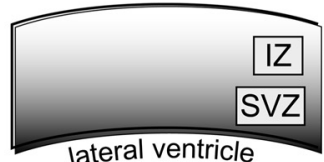

B

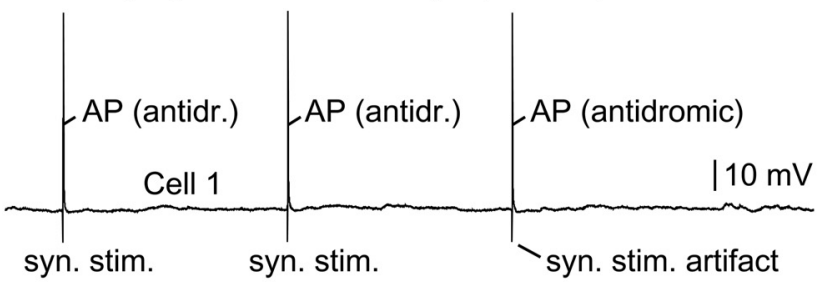

C Stimulation Outcome

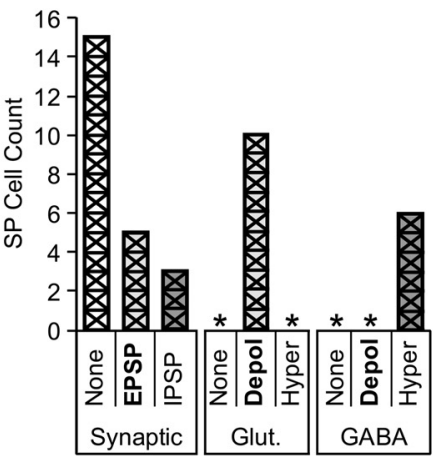

D

Synaptic Stimulation - Excitatory PSP

E

Synaptic Stimulation - Inhibitory PSP

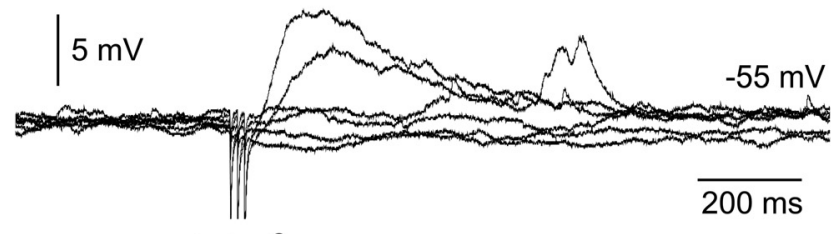

syn. $x 3$

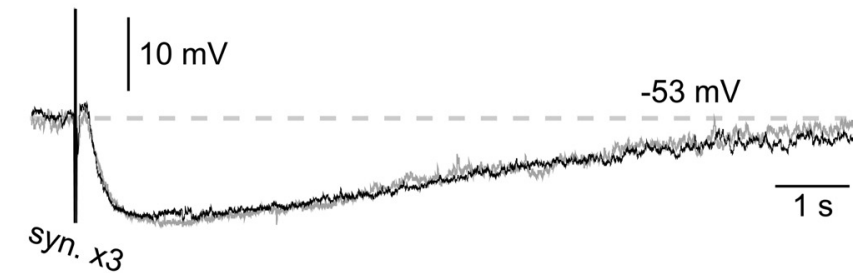

F Glutamatergic Stimulation (Microiontophoresis)
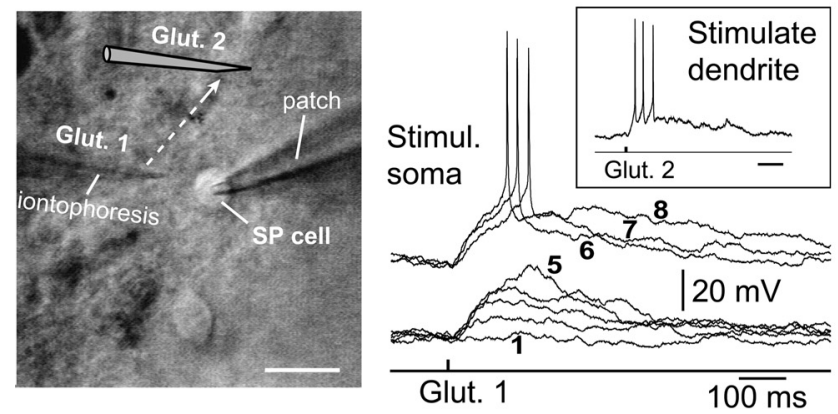

G

GABAergic Stimulation (Pressure Ejection)

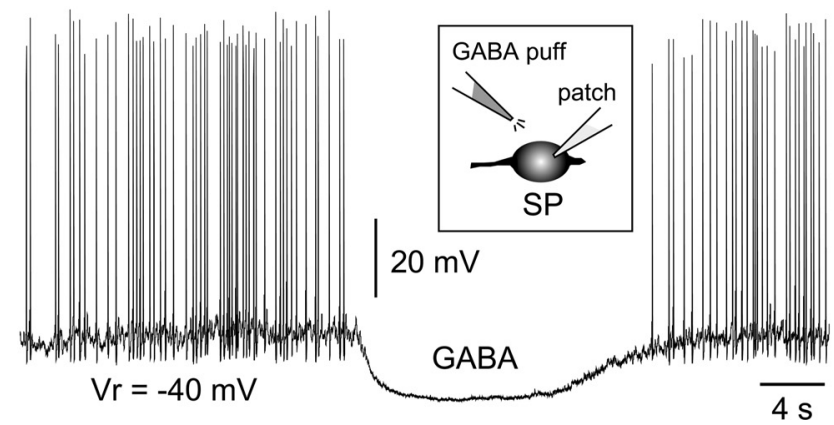

Figure 4. Synaptic physiology of human subplate neurons. $A$, Schematic of an acute brain slice. The synaptic stimulation electrode (syn. stim.) was moved between three locations: below, above, or lateral to the SP neuron. B, Failed responses to synaptic stimulation. C, Outcomes of the (1) synaptic shocks (Synaptic), (2) glutamate iontophoresis (Glut.), and (3) GABAergic applications (GABA) expressed by number of SP neurons that had no response (None), or experienced positive (EPSP; Depol.) or negative (IPSP; Hyper.) membrane potentials. Asterisks indicate zero count. $\boldsymbol{D}$, Synaptically evoked EPSP—-two successes, four failures. Three shocks were delivered at $50 \mathrm{~Hz}$ (syn. $\times 3$ ). E, Synaptically evoked IPSP -2 successes. Data are from a different cell with the same stimulation settings as in $\boldsymbol{D}$. $\boldsymbol{F}$, Left, During the experiment, the glutamate iontophoresis electrode was moved (dashed arrow) from the location near the soma (Glut. 1) to another position, near the apical dendrite (Glut. 2). The pia is toward top right. Scale bar, $20 \mu \mathrm{m}$. Right, Glutamate-evoked membrane potential transients-eight increments in iontophoretic current intensity, 1-8. Inset, Glutamate-evoked burst of APs. Calibration: $200 \mathrm{~ms}$. G, Spontaneous firing of APs is stopped by focal application of GABA. Inset, Experimental paradigm for local GABA application.

isting ultrastructural evidence for putative synaptic contacts on human SP neurons (Kostovic and Rakic, 1990) could be a scenario in which presynaptic fibers contact the SP neurons before ligand-gated receptor channels are expressed on the postsynaptic membrane. To determine whether human SP neurons express ligand-gated receptors in the next series of experiments, we use the exogenous amino acid neurotransmitters glutamate and GABA.

\section{Human SP neurons respond to glutamate and GABA}

Application of exogenous glutamate $(30-150 \mu \mathrm{m}$ from the cell body) elicited depolarizing membrane potentials in all neurons tested ( $n=10)$ (Fig. $4 F$, right). Gradually increasing intensities of iontophoretic current $(1.0 \mu \mathrm{A}$, increment $=0.1 \mu \mathrm{A})$ resulted in a gradual increase in the amplitude of evoked depolarizations (Fig. $4 F, 1-5)$, with AP firing of the SP neurons at higher iontophoretic current intensities (Fig. 4F,6-8). Application of glutamate at the dendrites of SP neurons also resulted in strong membrane depo- larizations (Fig. 4F, inset "Stimulate dendrite"). The glutamateevoked membrane response was robust and reliable, as it did not change with repetitive stimulation (Fig. $5 A, B$ ). In four SP neurons, glutamate pulses elicited sustained plateau-like depolarizations (Fig. 5C), which resembled synaptically evoked plateau potentials found in adult rodent cortex (Milojkovic et al., 2004). Therefore, human SP neurons begin to express functionally competent glutamate receptors on their plasma membrane as early as 20 gw.

To determine whether GABA receptors were present at these stages, we pressure applied exogenous GABA locally via glass pipette (Fig. 4G). Transient applications of GABA caused distinct and robust hyperpolarizations in all neurons tested $(n=6)$. The troughs of the GABA-induced hyperpolarizations varied around the mean value of $-63.1 \pm 2.2 \mathrm{mV}$. Analysis of the GABA reversal potential in current-clamp (Fig. 6A) and voltage-clamp (Fig. 6B) modes arrived at similar values (in the range between -75 and 


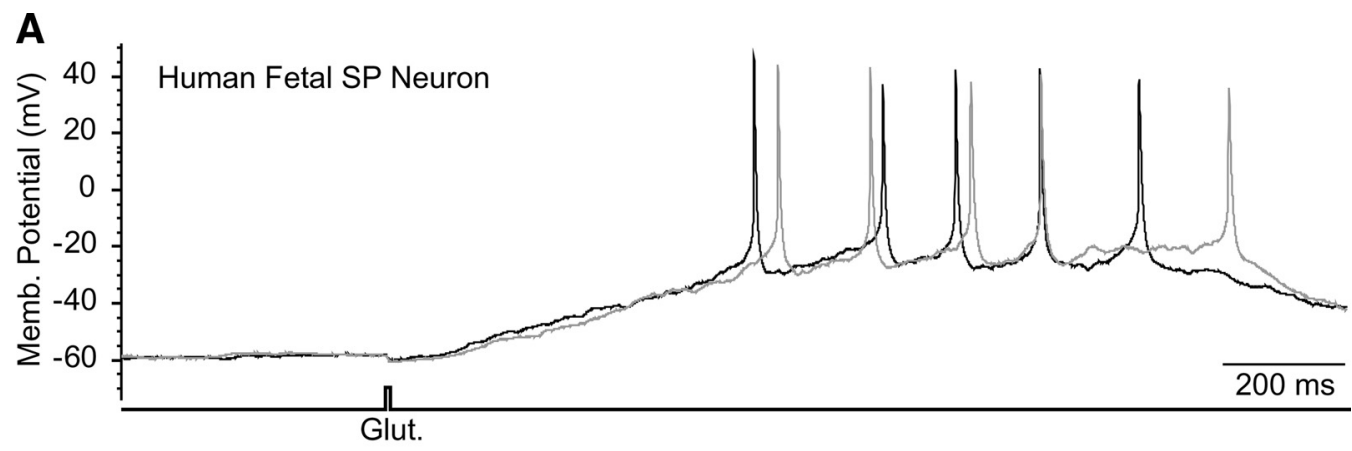

B Human Fetal SP Neuron

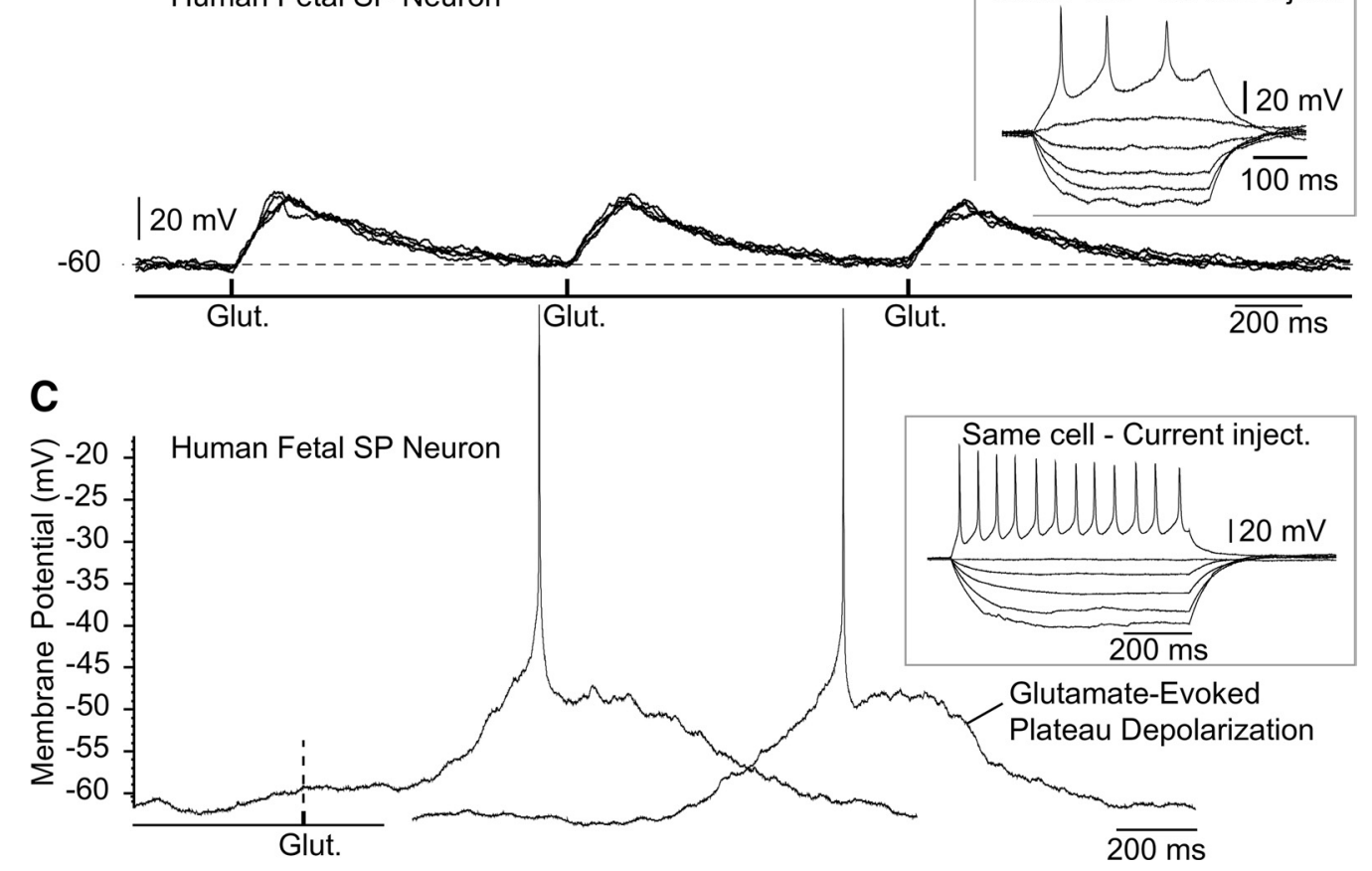

Figure 5. Glutamate-evoked responses in human SP neurons. A, SP neuron fires a burst of action potentials upon a single glutamatergic pulse (duration $5 \mathrm{~ms}$, intensity $=4 \mu \mathrm{A}$ ) delivered 150 $\mu \mathrm{m}$ away from the cell body. Glut. indicates the timing of the glutamate pulse. Two experimental trials (identical stimulation) are superimposed. $\boldsymbol{B}$, Subthreshold membrane potential transients in human SP neuron (21 gw) induced by three successive glutamate pulses (frequency $=1 \mathrm{~Hz}$; glutamate pulse duration $=5 \mathrm{~ms}$; intensity $=1.1 \mu \mathrm{A}$ ), repeated five times. All five recordings are superimposed. Inset, In the same cell, a somatic current injection reveals repetitive AP firing-a hallmark of the human SP neuron physiology. C, A single glutamatergic pulse (duration 5 ms, intensity $=3 \mu \mathrm{A}$ ) triggers a sustained depolarization of the SP neuron. Two experimental trials, same stimulation, are shown. Inset, Repetitive AP firing in the same cell.

$-60 \mathrm{mV})$. The GABA reversal potential is mostly determined by intracellular chloride ions. In animal models, during brain development the intracellular chloride concentration changes considerably, causing the change in GABAergic transmission from excitatory in immature to inhibitory in mature neurons (Ben-Ari, 2002). Our recordings were made in whole-cell configuration, which cannot preserve the physiological concentration of chloride ions inside the cell. Although our present experiments could not determine whether GABA is excitatory or inhibitory in the human cortex at this age, our present data unequivocally show that human SP neurons at midgestation express physiologically competent ionotropic GABA receptors on their membranes.

\section{Discussion}

Using postmortem human fetal tissue, we examined the propensity of early cortical neurons to generate spontaneous electrical activity. Only those cells that met all four anatomical and physiological criteria for SP neurons (as described in Results) were included in the present analysis.

\section{Electrical activity in human SP}

The majority of human SP neurons (25 of 49) exhibited spontaneous sustained depolarizations accompanied by firing of fullsize APs (Fig. 2) without any pharmacological manipulation. We used drug-free regular ACSF with $2 \mathrm{mM} \mathrm{Ca}^{2+}$ [but see SanchezVives and McCormick (2000) and Hanganu et al. (2009)]. The subplate UP states in human were stronger and richer than in rodent (Hanganu et al., 2009), probably because the human SP zone is severalfold thicker. Human SP zone contains a greater number of cellular elements, including neuronal cell bodies, dendrites, and axons (Valverde and Facal-Valverde, 1988; Kostovic and Rakic, 1990). The human SP zone may also possess a higher density of a particular subset of GABAergic SP neurons that propel network activity (Voigt et al., 2001).

\section{Scarce functional synaptic inputs}

In the majority of SP neurons (65\%), multiple stimulation sites and stimulation intensities (Fig. $4 A-C$ ) failed to uncover any signs of fast synaptic communication, despite clear evidence that 

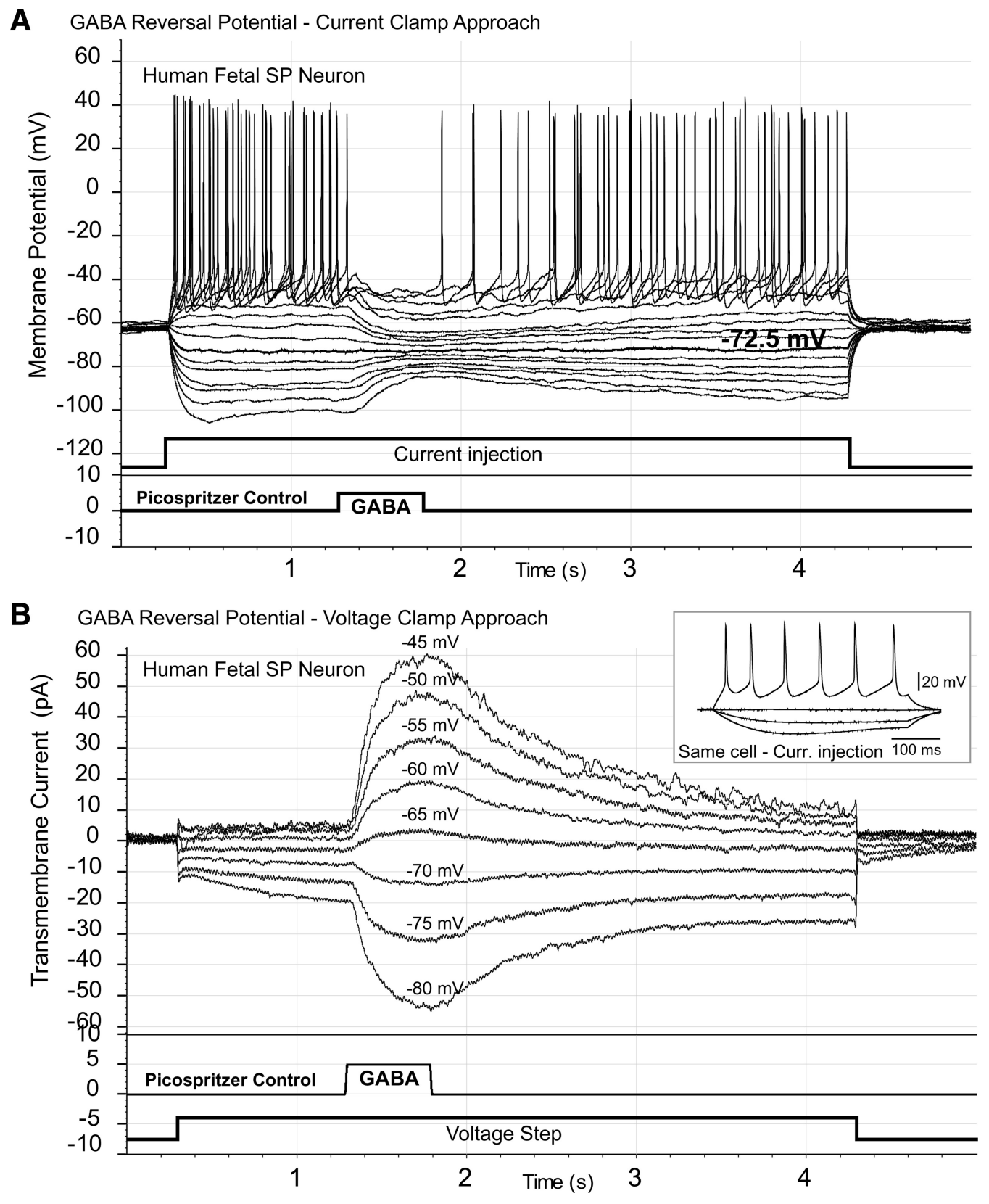

Figure 6. Reversal of the GABA-induced potential in human SP neurons. $A$, A set of 16 current pulses (current injection) was used to vary the membrane potential. Each time, a puff of GABA was delivered on the cell body using a computer-driven picospritzer. The GABA-induced postsynaptic potential reversed its polarity when the SP neuron was held at $-72.5 \mathrm{mV}$ (bold trace). $\boldsymbol{B}$, An SP neuron was voltage clamped at different voltages starting from $-80 \mathrm{mV}$, in $5 \mathrm{mV}$ increments (voltage step). At each voltage step, one GABA puff was locally applied. The reversal of GABA current polarity occurred between $-70 \mathrm{mV}$ and $-65 \mathrm{mV}$. Inset, This same cell fires repetitive APs upon direct current stimulation.

all human SP neurons readily respond to application of exogenous amino acids, glutamate and GABA (Fig. $4 F, G$ ). The most likely cause for an extremely low count of human SP neurons with electrophysiologically detectable synaptic contacts may lie in the age $(20-21 \mathrm{gw})$ used in the present study. There is an uncertainty about the number of glutamatergic and GABAergic axons in the human SP at 20 and $21 \mathrm{gw}$. The fibers carrying glutamate and GABA into the human SP are clearly preceded by monoaminergic projections between 12 and $15 \mathrm{gw}$ (Zecevic and Verney, 1995), followed by cholinergic afferents between 15 and 18 gw (Kostovic and Rakic, 1984). The glutamate-releasing thalamocortical and corticocortical connections arrive last (Kostovic and Goldman-Rakic, 1983); thus, only a few of them may have reached their target at the fetal age analyzed in the present study
(20-21 gw). It is possible that at $20-21 \mathrm{gw}$, the few ascending thalamocortical afferents that have reached the SP zone have not yet made direct synaptic contacts (Hevner, 2000), but rather engage in nonsynaptic forms of communication (i.e., volume transmission), as reported for GABAergic transmission in the rodent SP (Hanganu et al., 2009). Based on our present electrophysiological data acquired in human occipital cortex at gestational ages 20 and 21 gw, the physiologically competent glutamate- and GABAreleasing afferents to $\mathrm{SP}$ neurons are quite rare (Fig. $4 C-E$ ).

\section{Early cortical UP states}

Spontaneous outbursts of network activity, cortical UP states, have been described in vivo under anesthesia (Cowan and Wilson, 1994) and during normal sleep (Steriade et al., 2001). Here, we provide 
evidence that this characteristic cortical signal (burst of APs superimposed on top of a plateau depolarization) also characterizes the spontaneous electrical activity of human SP neurons (Figs. 2, 3; supplemental Figs. S1, S2, available at www.jneurosci.org as supplemental material) 4 months before birth (20-21 gw).

Early spontaneous electrical activity is thought to guide the most fundamental processes of nervous system development, including neuronal migration, axonal outgrowth, transmitter phenotype selection, dendritic patterning, activation of transmitter receptors, programmed cell death, and the development of mature ion channel types (Moody and Bosma, 2005; Spitzer, 2006). In respect to the region of origin (cortex) and electrical pattern (i.e., amplitude, duration, and frequency), the spontaneous electrical activity found in human SP cells resembles some aspects of cortical slow-wave oscillations (Cowan and Wilson, 1994; Chauvette et al., 2010). Just like adult cortical pyramidal neurons, human fetal SP neurons spend time in both quiet DOWN states and active UP states (Fig. 2A). The greatest difference between fetal and adult UP states lies in the rhythm. The depolarizing events in human fetal cerebral cortex occur sporadically and often after long periods of silence (Fig. $2 \mathrm{~A}$, Silent period). The first notion of the discontinuous nature of the early cortical activity came from EEG recordings obtained in premature neonates, and thus termed "trace discontinue" (Lamblin et al., 1999). Based on a discontinued nature of the fetal activity in vitro (Fig. $2 A$ ) one is tempted to propose that "trace discontinue EEG" could be a product of the thick human SP. Recall that at this age, human SP zone is $4-5$ times wider than the human $\mathrm{CP}$ (Fig. $1 A$ ). With large volume, mature membrane properties of SP neurons (Moore et al., 2009), and the propensity of human SP neurons to generate spontaneous electrical events in vitro (present data), it is possible that the EEG signal in preterm infants largely reflects the physiological function of the SP zone.

The functional role of the fetal cortical UP states is unknown. The unprovoked outbursts of electrical discharge (UP states) shown in Figures 2 and 3 may be involved in the establishment of the human cortical network before formation of functional thalamocortical synapses. In light of the present data, cortical electrical oscillations seem relevant not only for maintenance and memory consolidation of mature neuronal networks (Marshall et al., 2006), but also in the construction of cortical circuitry before birth (Moody and Bosma, 2005; Luhmann et al., 2009). We think that the cortical oscillatory rhythm starts as unsynchronized slow events before birth (Fig. 2; supplemental Figs. S1, S2, available at www.jneurosci.org as supplemental material) and then continues as synchronized oscillatory patterns throughout life, whenever sensory inputs cease to impinge onto the cerebral cortex (during deep sleep).

\section{References}

Allène C, Cattani A, Ackman JB, Bonifazi P, Aniksztejn L, Ben-Ari Y, Cossart R (2008) Sequential generation of two distinct synapse-driven network patterns in developing neocortex. J Neurosci 28:12851-12863.

André M, Lamblin MD, d'Allest AM, Curzi-Dascalova L, Moussalli-Salefranque F, Nguyen The Tich S, Vecchierini-Blineau MF, Wallois F, Walls-Esquivel E, Plouin P (2010) Electroencephalography in premature and full-term infants. Developmental features and glossary. Neurophysiol Clin 40:59-124.

Ben-Ari Y (2002) Excitatory actions of gaba during development: the nature of the nurture. Nat Rev Neurosci 3:728-739.

Chauvette S, Volgushev M, Timofeev I (2010) Origin of active states in local neocortical networks during slow sleep oscillation. Cereb Cortex 20:2660-2674.

Corlew R, Bosma MM, Moody WJ (2004) Spontaneous, synchronous electrical activity in neonatal mouse cortical neurones. J Physiol 560:377-390.

Cowan RL, Wilson CJ (1994) Spontaneous firing patterns and axonal projections of single corticostriatal neurons in the rat medial agranular cortex. J Neurophysiol 71:17-32.
Garaschuk O, Linn J, Eilers J, Konnerth A (2000) Large-scale oscillatory calcium waves in the immature cortex. Nat Neurosci 3:452-459.

Ghosh A, Antonini A, McConnell SK, Shatz CJ (1990) Requirement for subplate neurons in the formation of thalamocortical connections. Nature 347:179-181.

Hanganu IL, Okabe A, Lessmann V, Luhmann HJ (2009) Cellular mechanisms of subplate-driven and cholinergic input-dependent network activity in the neonatal rat somatosensory cortex. Cereb Cortex 19:89-105.

Hevner RF (2000) Development of connections in the human visual system during fetal mid-gestation: a DiI-tracing study. J Neuropathol Exp Neurol 59:385-392.

Kostovic I, Goldman-Rakic PS (1983) Transient cholinesterase staining in the mediodorsal nucleus of the thalamus and its connections in the developing human and monkey brain. J Comp Neurol 219:431-447.

Kostovic I, Rakic P (1984) Development of prestriate visual projections in the monkey and human fetal cerebrum revealed by transient cholinesterase staining. J Neurosci 4:25-42.

Kostovic I, Rakic P (1990) Developmental history of the transient subplate zone in the visual and somatosensory cortex of the macaque monkey and human brain. J Comp Neurol 297:441-470.

Lamblin MD, Andre M, Challamel MJ, Curzi-Dascalova L, d'Allest AM, De Giovanni E, Moussalli-Salefranque F, Navelet Y, Plouin P, RadvanyiBouvet MF, Samson-Dollfus D, Vecchierini-Blineau MF (1999) [Electroencephalography of the premature and term newborn. Maturational aspects and glossary] Neurophysiol Clin 29:123-219.

Luhmann HJ, Kilb W, Hanganu-Opatz IL (2009) Subplate cells: amplifiers of neuronal activity in the developing cerebral cortex. Front Neuroanat 3:19.

Luskin MB, Shatz CJ (1985) Studies of the earliest generated cells of the cat's visual cortex: cogeneration of subplate and marginal zones. J Neurosci 5:1062-1075.

Marshall L, Helgadóttir H, Mölle M, Born J (2006) Boosting slow oscillations during sleep potentiates memory. Nature 444:610-613.

Milojkovic BA, Radojicic MS, Goldman-Rakic PS, Antic SD (2004) Burst generation in rat pyramidal neurones by regenerative potentials elicited in a restricted part of the basilar dendritic tree. J Physiol 558:193-211.

Moody WJ, Bosma MM (2005) Ion channel development, spontaneous activity, and activity-dependent development in nerve and muscle cells. Physiol Rev 85:883-941.

Moore AR, Filipovic R, Mo Z, Rasband MN, Zecevic N, Antic SD (2009) Electrical excitability of early neurons in the human cerebral cortex during the second trimester of gestation. Cereb Cortex 19:1795-1805.

Rakic P (1976) Prenatal genesis of connections subserving ocular dominance in the rhesus monkey. Nature 261:467-471.

Rakic S, Zecevic N (2000) Programmed cell death in the developing human telencephalon. Eur J Neurosci 12:2721-2734.

Sanchez-Vives MV, McCormick DA (2000) Cellular and network mechanisms of rhythmic recurrent activity in neocortex. Nat Neurosci 3:1027-1034.

Spitzer NC (2006) Electrical activity in early neuronal development. Nature 444:707-712.

Steriade M, Timofeev I, Grenier F (2001) Natural waking and sleep states: a view from inside neocortical neurons. J Neurophysiol 85:1969-1985.

Stern EA, Kincaid AE, Wilson CJ (1997) Spontaneous subthreshold membrane potential fluctuations and action potential variability of rat corticostriatal and striatal neurons in vivo. J Neurophysiol 77:1697-1715.

Valverde F, Facal-Valverde MV (1988) Postnatal development of interstitial (subplate) cells in the white matter of the temporal cortex of kittens: a correlated Golgi and electron microscopic study. J Comp Neurol 269:168-192.

Voigt T, Opitz T, de Lima AD (2001) Synchronous oscillatory activity in immature cortical network is driven by GABAergic preplate neurons. J Neurosci 21:8895-8905.

Xia Y, Haddad GG (1999) Effect of prolonged O2 deprivation on $\mathrm{Na}+$ channels: differential regulation in adult versus fetal rat brain. Neuroscience 94:1231-1243.

Yuste R, Peinado A, Katz LC (1992) Neuronal domains in developing neocortex. Science 257:665-669.

Zecevic N, Verney C (1995) Development of the catecholamine neurons in human embryos and fetuses, with special emphasis on the innervation of the cerebral cortex. J Comp Neurol 351:509-535.

Zhang JH, Gibney GT, Xia Y (2001) Effect of prolonged hypoxia on $\mathrm{Na}+$ channel mRNA subtypes in the developing rat cortex. Brain Res Mol Brain Res 91:154-158. 